\title{
Analysis and Motion Control of a Centrifugal-Force Microrobotic Platform
}

\author{
Panagiotis Vartholomeos, Member, IEEE, Kostas Vlachos, and Evangelos Papadopoulos, Senior Member, IEEE
}

\begin{abstract}
This paper presents the analysis, design, and closedloop motion control of a mobile microrobotic platform capable of micrometer positioning on a plane. The mobile microrobot, including chassis, actuators, drives, microprocessor, and electronics, is of low cost (less than \$20), can be fabricated rapidly and is made of commercially available components. Its motion is induced by centrifugal forces generated by two vibration motors installed inside the platform body. The asynchronous operation of the vibration motors is shown by simulation to result in planar motions of two degrees-of-freedom locally, with micrometer resolution. A motion controller has been designed to generate controlled motions using sets of motor angular velocities. A prototype has been developed and used to validate the motion principle and the controller efficacy. Open loop experiments show that the platform motion resolution is approximately $20 \mu \mathrm{m}$, while its speed is greater than $2 \mathrm{~mm} / \mathrm{s}$. Closed-loop experiments demonstrate a $5 \mu \mathrm{m}$ resolution, i.e., a fourfold improvement compared to the open loop experiments. The low cost, the rapid fabrication, and the micrometer motion resolution suggest that this microrobotic platform is a promising solution for low-cost microfactories, where a group of such robots performs high throughput, advanced microassembly of microsystems.
\end{abstract}

Note to Practitioners-The aim of this work is to increase the level of autonomy and motion flexibility in micromanipulation and microassembly tasks. Despite their nanometer resolution, current micromanipulation platforms either occupy extreme volumes with respect to their workspace or suffer from expensive and/or bulky power and driving units that limit their effectiveness in complex cooperative tasks. The developed system is a low-cost, tetheredless, fully autonomous, microrobotic platform that can perform micromanipulation and microassembly tasks, such as the cooperative fabrication of microsystems or manipulation of biological specimens, in a micro scale environment. The platform motion principle exploits the centrifugal forces generated by DC vibration motors installed in the body of a microrobot, that moves as cell phones move when they vibrate. Experiments showed that the closed-loop motion resolution is $5 \mu \mathrm{m}$. A group of such robots can be used in cooperative micromanipulation and/or microassembly tasks in the micrometer scale.

Index Terms-Microassembly, microrobotics, mobile robot, motion control, vibration motor.

Manuscript received June 15, 2012; revised October 05, 2012; accepted January 19, 2013. This paper was recommended for publication by Associate Editor P. Lambert and Editor XXXXXXX upon evaluation of the reviewers' comments.

P. Vartholomeos is with Children's Hospital Boston, Boston, MA 02115 USA, and Harvard Medical School, Boston, MA 02115 USA (e-mail: Panagiotis.Vartholomeos@childrens.harvard.edu).

K. Vlachos and E. Papadopoulos are with the Department of Mechanical Engineering, National Technical University of Athens, 15780 Athens, Greece (e-mail: kostaswl@central.ntua.gr; egpapado@central.ntua.gr).

Color versions of one or more of the figures in this paper are available online at http://ieeexplore.ieee.org.

Digital Object Identifier 10.1109/TASE.2013.2248083

\section{INTRODUCTION}

$\mathbf{P}$ ACKAGING and assembly cost is a major portion of the overall cost of microsystems technologies (MSTs), especially of those involving many heterogeneous parts [1]. To reduce these costs, extensive research has been carried out on the design and development of robotic manipulators that can fabricate MSTs more efficiently and at a higher throughput. The fabrication tasks require robots of micron motion resolution. These are called microrobots; a name attributed to their high motion resolution and not to their actual size, which typically lies on the $\mathrm{cm}^{3}$ scale to be able to impart forces sufficiently high for microassembly tasks.

Currently, MSTs are fabricated using microrobots that are bolted at specific locations along an assembly line performing manipulations of nanometer resolution, at high speeds, with excellent motion stability and robustness [2]. A disadvantage of these microrobots is that they occupy a large volume compared to their small reachable workspace. Their large size in addition to the structured environment of the assembly line result in a system of reduced flexibility that cannot perform tasks of high complexity. This limitation can be addressed by a system that exhibits a small footprint and at the same time provides the ability to restructure the workspace depending the nature of the task. These requirements can be met by a group of tetheredless, miniature, mobile, microinstrumented, cooperative microrobots, which combine micron-resolution capabilities together with virtually unlimited workspace [3].

The key component of miniature mobile microrobots is their actuation mechanism, because it is directly related to the micron-resolution capabilities, the power autonomy, and the compact design [4]. The most popular micro-positioning motion mechanism is the stick-slip principle, which is implemented using piezoelectric actuators [3]-[8]. This principle is employed by the MINIMAN micro-robot [3], and by the MiCRoN robots [5], [8]. Although piezoelectric actuators do provide the required positioning resolution, they usually suffer from expensive and bulky power and driving units. Therefore, it is difficult to develop a low-cost, tetheredless, autonomous platform. Small-scale piezoelectric drivers and amplifiers that could be accommodated on board are custom made and thus do not allow for cost effective designs [9].

The limitations of the microrobots in [3]-[8] have been overcome by a novel actuation method first presented in [10], [11]. This actuation method employs centrifugal forces generated by DC vibration micromotors installed inside the body of the microrobot. This approach results in compact, low-cost, easily fabricated microrobotic platforms. The scalability of these systems 


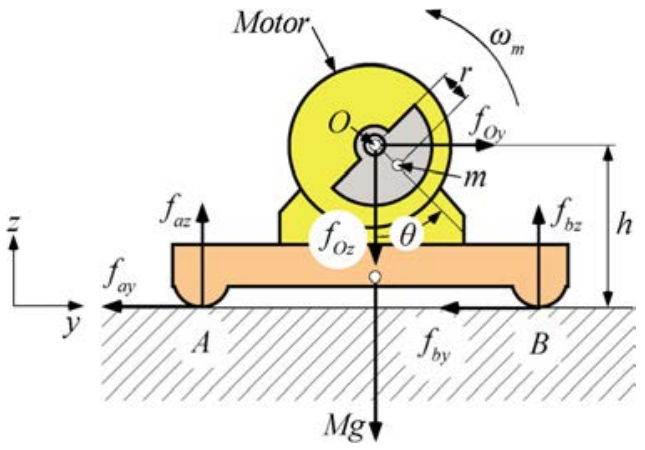

Fig. 1. Simplified one dof platform with rotating mass $m$.

and their suitability for collective behaviors has been demonstrated in [12].

In this paper, we demonstrate for the first time a complete analysis of the dynamics of the robotic platform presented in [10] and [11] and we demonstrate for the first time closed-loop motion control experiments. More specifically, a summary of the underlying physics of the actuation principle is presented in Section II and the platform dynamics in Section III. In Section IV, a brief description of the synchronous operation is given, and the asynchronous operation of the DC vibration motors is analyzed and is used to generate the two degree of freedom (two dof) planar motion of the platform. The proposed controller, and the first microrobot prototype are described in Sections V and VI, respectively. Open loop and closed-loop motion control experiments are conducted and evaluated in Sections VII and VIII, respectively, where their comparison showed that closed-loop control results in $5 \mu \mathrm{m}$ motion resolution, i.e., a fourfold improvement compared to the open loop control operation.

\section{MOTION PRINCIPLE}

The underlying physics of the actuation mechanism are explained using a simplified one degree of freedom (one dof) mobile platform of mass $M$. The actuation mechanism employs an eccentric mass $m$, rotated at a constant angular speed $\omega_{m}$ by a platform-mounted motor, as shown in Fig. 1.

The actuation angle $\theta$ defines the angular position of the eccentric mass $m$ with respect to the vertical axis, see Fig. 1. One cycle of operation is completed when the mass $m$ has described an angle of $360^{\circ}$. Gravitational and centripetal forces exerted on the rotating mass are resolved along the $y-z z$ axes to yield

$$
\begin{aligned}
& f_{O y}=m r \omega_{m}^{2} \sin \theta \\
& f_{O z}=-m g-m r \omega_{m}^{2} \cos \theta
\end{aligned}
$$

where $g$ is the acceleration of gravity and $r$ is the arm of eccentricity of $m$ with respect to $O$. These forces are transmitted to the platform at point $O$, while the small moment due to $m$ is neglected. When the angular speed $\omega_{m}$ is low, the platform does not move because the horizontal actuation force $f_{O y}$ is cancelled by frictional forces at the platform contact points A and $\mathrm{B}$. However, if the angular speed $\omega_{m}$ exceeds a critical value $\omega_{m_{-} \text {critical }}$, then $f_{O y}$ overcomes the support point friction forces, and as a result, the platform begins to slide.
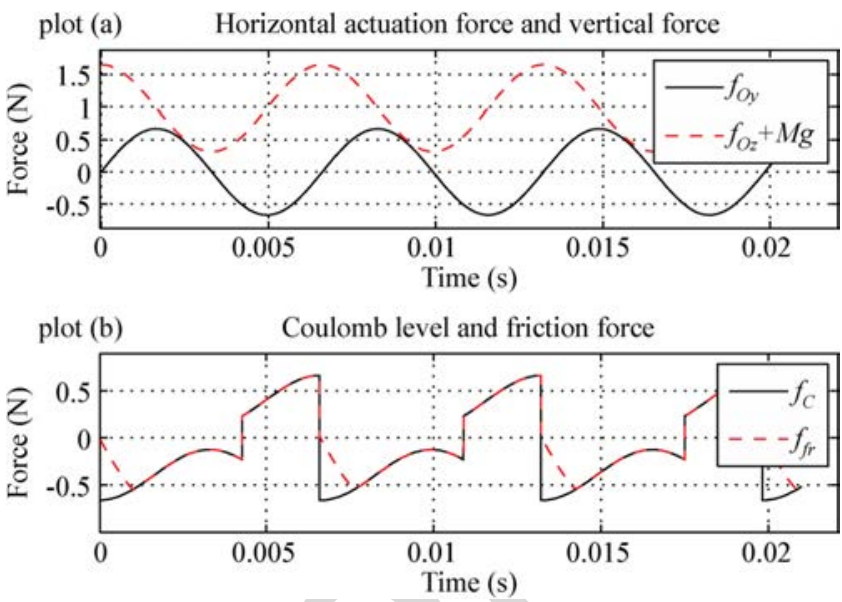

Fig. 2. (a) Horizontal and (b) vertical forces acting on a one dof platform.

Using a simplified static-kinetic friction model, the motion of the platform along the $y$ and $z$ axes is described by

$$
\begin{aligned}
M \ddot{y} & =f_{O y}-f_{f r} \\
0 & =f_{a z}+f_{b z}+\left(-M g+f_{O z}\right)
\end{aligned}
$$

where all forces are defined in Fig. 1 , and $f_{f r}$ is the friction force. Neglecting viscous friction, $f_{f r}$ is given by

$$
f_{f r}=\left\{\begin{array}{lll}
f_{c} \operatorname{sgn}(\dot{y}), & y \neq 0 \\
f_{O y}, & \left\|f_{O y}\right\|<f_{c}, \quad \dot{y}=0, & \ddot{y}=0 \\
f_{c} \operatorname{sgn}\left(f_{O y}\right), & \left\|f_{O y}\right\|>f_{c}, \quad \dot{y}=0, \quad \ddot{y} \neq 0
\end{array}\right.
$$

where $f_{C}$ is the Coulomb friction level, i.e. the maximum friction force that can exist for the current normal force, and is given by

$$
f_{c}=\mu\left(f_{a z}+f_{b z}\right)=\mu\left(M g=f_{O z}\right) .
$$

The parameter $\mu$ is the coefficient of kinetic friction and the function $\operatorname{sgn}(\dot{y})$ is defined by

$$
\operatorname{sgn}(\dot{y})=\left\{\begin{array}{cl}
+1, & \dot{y}>0 \\
0, & \dot{y}=0 . \\
-1, & \dot{y}<0
\end{array}\right.
$$

The forces acting on the platform are given by (1), (3), and (4) and are plotted in Fig. 2 for three consecutive cycles.

It is observed that the horizontal actuation force $f_{O y}$ and the vertical actuation force $f_{O z}$ are time periodic and $f_{O z}$ leads $f_{O y}$ by $\pi / 2$ [Fig. 2(a)].

Due to (4), the Coulomb friction level $f_{C}$ is periodic too and in phase with $f_{O z}$, but its sign changes from positive to negative depending on the speed direction [Fig. 2(b)]. This figure also shows the friction force $f_{f r}$. The platform's motion response caused by the forces in Fig. 2 is computed by numerical integration of (2) and is presented in Fig. 3.

The physics of the motion principle are explained next in more detail. Due to (1), when the actuation angle $\theta$ is small, the actuation force $f_{O y}$ is not sufficient to overcome the Coulomb level and no motion is induced. At a critical angle $\theta_{1}$, the actuation force $f_{O y}$ overcomes the static friction limit $f_{C}$, and motion is induced (Fig. 3). The platform executes forward motion. 


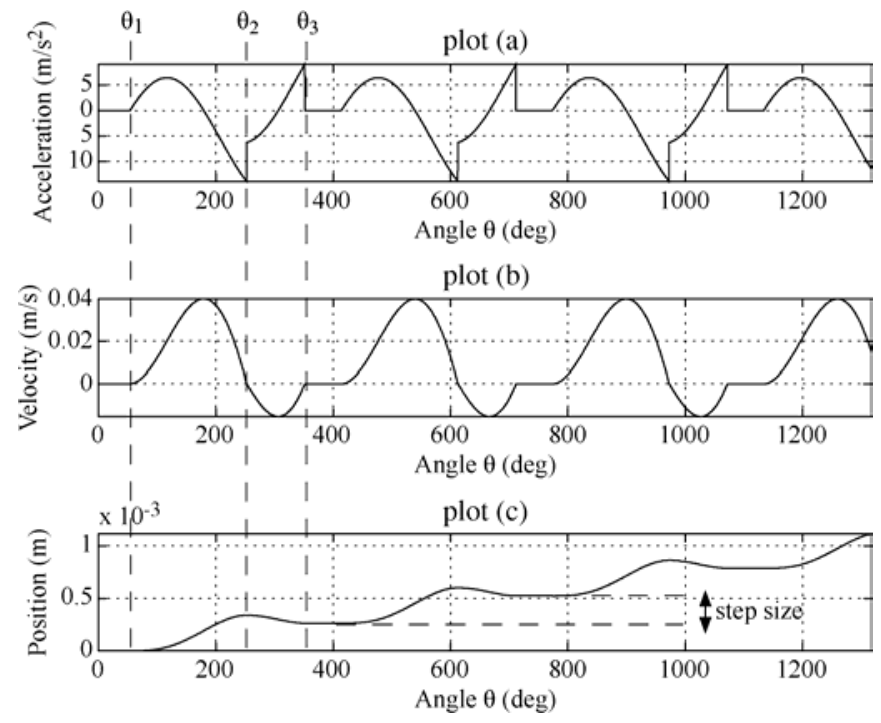

Fig. 3. Platform motion: (a) acceleration, (b) velocity, and (c) position corresponding to the forces of Fig. 2.

When $m$ passes the highest point at $\theta=180^{\circ}$, the platform already has a positive velocity. As $m$ moves past this point, friction forces together with actuation forces decelerate the platform. As friction still increases, it eventually brings the platform to a stop at a critical angle $\theta_{2}$ (Fig. 3). The actuation forces are now pointing to the left and as a result reverse platform motion starts. While $m$ rotates to the forth quadrant of the actuation cycle, the reverse platform motion decelerates and eventually stops at critical angle $\theta_{3}$ (Fig. 3).

Quite interestingly, as shown in Fig. 3(c), for a counterclockwise rotation of the eccentric load, the platform exhibits a net displacement along the positive $y$ axis. This is due to the fact that during platform forward motion, the eccentric mass is at the higher points of its trajectory (second quadrant of actuation cycle) and, therefore, the normal forces and the frictional forces are low, whereas during the reverse motion, the mass is at the lower points of its trajectory (fourth quadrant of actuation cycle) and the frictional forces are high. Consequently, the platform decelerates more during reverse motion compared to forward motion and therefore - for a counterclockwise rotation of the eccentric load - a net displacement towards the positive $y$ axis takes place.

\section{PlatForm DyNAMics}

Two centrifugal force actuators, are employed in the design of a microrobotic platform capable of two dof planar motions, see Fig. 4. Although it is easier to drive the microrobot using more actuators, in the sense that there is no need for a motion planning algorithm, it would be less efficient. When more motors are used some of the components of the horizontal actuation forces cancel out each other (a null-space is generated) and consequently efficiency is reduced. Furthermore, having more motors reduces significantly the capability for miniaturization, increase the cost, and the complexity of the design.

Platform base: The contact points between the platform and the ground are provided by three fixed small steel balls A, B, and $\mathrm{C}$ located at the vertices of an equilateral triangle [Fig. 4(b)].
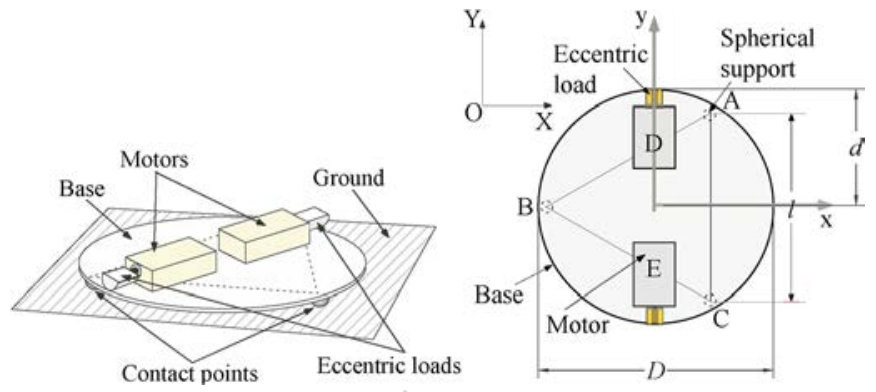

Fig. 4. The two-actuator platform concept: (a) angle view and (b) top view.

The length between the ball supports is $l$, while the radius of the platform base is $d$ [Fig. 4(b)]. The three-contact point configuration is favored because it is not overconstrained and ensures static equilibrium along the vertical axis.

Actuators: The actuation of the platform employs miniaturevibrating motors. Each vibrating motor is axially coupled to an eccentric load, while the control input is the rotation speed $\omega_{m}$ of the motor. During motor rotation, the eccentric mass of the load generates periodic dynamic forces, which are transferred to the contact points and interact with the friction forces.

\section{A. Platform Dynamics}

Using the Newton-Euler formulation, the platform dynamics are described by [13]

$$
\begin{aligned}
M \dot{\mathbf{v}} & =\mathbf{R} \sum_{i}{ }^{b} \mathbf{f}_{i}, \quad i=\{a, b, c, d, e\} \\
I_{z z} \ddot{\psi} & =\hat{\mathbf{z}} \sum_{i}\left({ }^{b} \mathbf{r}_{i} \times{ }^{b} \mathbf{f}_{i}\right), \quad i=\{a, b, c, d, e\}
\end{aligned}
$$

where $b$ is the body-fixed frame, $\mathbf{R}$ is the rotation matrix between frame $b$ and the inertial frame $O$ [see Fig. 4(b)], $\psi$ is the platform angle of rotation, and $\mathbf{v}=[\dot{x}, \dot{y}, \dot{z}]^{T}$ is its center of mass $(\mathrm{CM})$ velocity with respect to the inertial frame $O$. In (7), $I_{z z}$ is the polar moment of inertia in the body fixed frame and $\hat{\mathbf{z}}$ denotes the unit $z$ axis vector. In both equations, the subscripts $i=\{a, b, c\}$ correspond to frictional forces at the contact points of the platform, and $i=\{d, e\}$ correspond to the forces generated by the two vibrating motors. The actuation forces that act on the platform, when the DC micromotors rotate (assuming identical micromotors), are given by

$$
\begin{aligned}
{ }^{b} f_{i x} & =-m r \dot{\theta}_{i}^{2} \sin \theta_{i} \\
{ }^{b} f_{i z} & =-m g_{i}-m r \dot{\theta}_{i}^{2} \cos \theta_{i}
\end{aligned}
$$

where $i=\{d, e\}$ and $\theta_{i}$ is the angle of micromotor $i, m$ is the micromotor eccentric mass, and $r$ is the arm of eccentricity. The dynamics of the DC micromotor $i$ are given by

$$
\begin{aligned}
\ddot{\theta}_{i} & =-\frac{b}{J} \dot{\theta}_{i}+\frac{k_{t}}{J} i_{L i}-\frac{m g r \sin \theta_{i}}{J}-\frac{c}{J} \\
\dot{i}_{L i} & =-\frac{k_{t}}{L} \dot{\theta}_{i}-\frac{R}{L} i_{L i}+\frac{1}{L} V_{i n_{-i}}
\end{aligned}
$$

where $i_{L i}$ is the motor $i$ current, $R$ is the electrical resistance, $b$ is the viscous friction, $c$ is the Coulomb friction at the micromotor's axis, $k_{t}$ is the torque constant, $L$ is the inductance, 


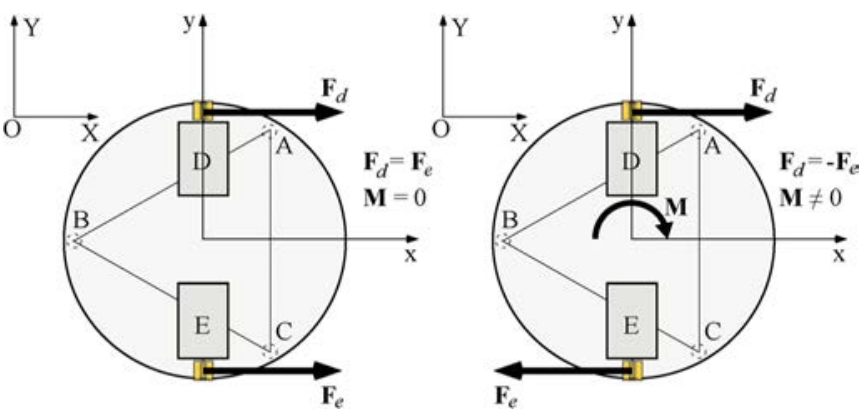

Fig. 5. Synchronous actuation: (a) pure linear and (b) pure rotational motion.

$J$ is the eccentric's load moment of inertia, and $V_{i n_{-} i}$ the input voltage of motor $i$.

\section{Platform Driving Modes}

When actuators are operating at the same rotational speed, then their operation is called synchronous, otherwise it is called asynchronous. When actuators operate synchronously the problem reduces to the cases depicted in Fig. 5. In the first case, $\omega_{m d}=\omega_{m e}=\omega_{m}$ and $\theta_{d}=\theta_{e}=\theta$, where $\omega_{m i}, i=\{d, e\}$ are angular actuation speeds of motors $\mathrm{D}$ and $\mathrm{E}$, respectively. As a result, the centrifugal forces $\mathbf{F}_{d}$ and $\mathbf{F}_{e}$ are equal and the platform performs pure linear motion. In the second case, the motors rotate at an opposite sense, and the platform performs pure rotational motion.

Synchronous actuation generates three distinct motion states of the platform, depending on the magnitude of the actuation forces. These are the following.

1) Static state: The actuation speed $\omega_{m}$ is smaller than a threshold value $\omega_{m_{-} s l}$ and the resulting actuation forces are not sufficient to overcome the frictional forces at the contact points. No motion is induced.

2) Sliding state: The actuation speed $\omega_{m}$ is greater than the threshold value $\omega_{m_{-} s l}$. Actuation forces exceed the Coulomb friction level, and consequently slip of the platform occurs and motion (sliding) is induced.

3) Tipping: The actuation speed $\omega_{m}$ is greater than a threshold value $\omega_{m_{-} t i p}$. The resulting actuation forces cause loss of static equilibrium along the vertical axis and tip of the platform occurs.

The three distinct motion states are delimited by the threshold actuation values $\omega_{m_{-s l}}$, and $\omega_{m_{-} t i p}$. These have been derived analytically, see [10], and are summarized in Table I and define the useful actuation range $\omega^{*}$, where $\omega_{m_{-} s l}<\omega^{*}<\omega_{m_{-} t i p}$ always drives the platform in the sliding mode.

When actuators $D$ and $E$ operate asynchronously, i.e., at rotational speeds $\omega_{m d}, \omega_{m e}$, where $\omega_{m d} \neq \omega_{m e}$, and assuming $\omega_{m d}, \omega_{m e}$ are constant, then the resultant actuation forces are the superposition of sinusoids of different magnitude and different frequency and form sinusoidal beats

$$
\begin{aligned}
& f_{x}(t)=A_{d} \sin \left(\omega_{m d} t\right)-A_{e} \sin \left(\omega_{m e} t\right) \\
& f_{z}(t)=-2 m g-\left(A_{d} \cos \left(\omega_{m d} t\right)+A_{e} \cos \left(\omega_{m e} t\right)\right)
\end{aligned}
$$

TABLE I

Threshold VALUes of SyNChronous ActuATION SPEED

\begin{tabular}{|c|c|c|}
\hline & Translation & Rotation \\
\hline$\omega_{m_{-} s l}$ & $\sqrt{\frac{\mu g M}{2 m r \sqrt{1+\mu^{2}}}}$ & $\sqrt{\frac{\mu g M}{2 m r \sqrt{\mu^{2}+\left(\frac{d}{\sqrt{2 l}}\right)^{2}}}}$ \\
\hline$\omega_{m_{-} \text {tip }}$ & $\sqrt{\frac{g M}{2 m r \sqrt{1+\left(\frac{h}{\sqrt{2 l}}\right)^{2}}}}$ & $\sqrt{\frac{g M}{2 m r}}$ \\
\hline
\end{tabular}
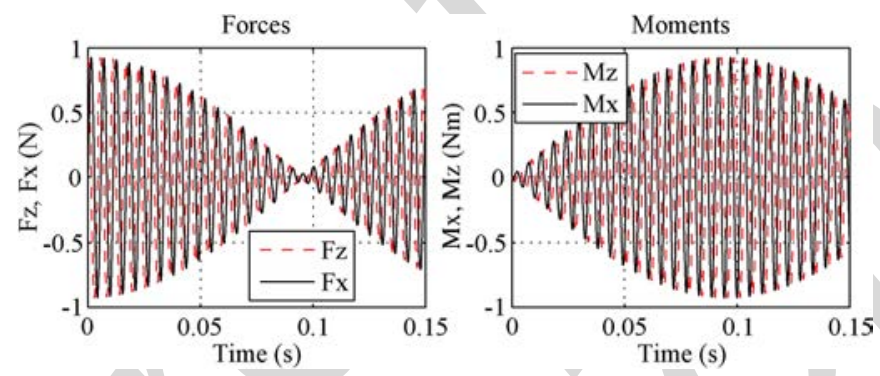

Fig. 6. Sinusoidal beat waveform: (a) actuation forces and (b) actuation moments.

Moments are generated about the $z$ and $x$ axes as described by

$$
\begin{aligned}
& M_{z}(t)=-d A_{d} \sin \left(\omega_{m d} t\right)+d A_{e} \sin \left(\omega_{m e} t\right) \\
& M_{x}(t)=d A_{d} \cos \left(\omega_{m d} t\right)-d A_{e} \cos \left(\omega_{m e} t\right)
\end{aligned}
$$

where $A_{d}=m_{d} r_{d} \omega_{m d}^{2}, A_{e}=m_{e} r_{e} \omega_{m e}^{2}$. The corresponding actuation forces and moments are depicted in Fig. 6.

The asynchronous operation of the platform is demonstrated through two simulation examples. In the first example, $\omega_{m d}=$ $1080 \mathrm{rad} / \mathrm{s}$ and $\omega_{m e}=900 \mathrm{rad} / \mathrm{s}$, while in the second $\omega_{m d}=$ $1060 \mathrm{rad} / \mathrm{s}$ and $\omega_{m e}=950 \mathrm{rad} / \mathrm{s}$. Both actuation sets lie within the useful actuation range $\omega^{*}$, which in the case of asynchronous operation is determined numerically. The $x, y, \psi$ motion responses are presented in Fig. 7. All three plots superimpose the motion generated by the two simulation sets. The first plot depicts the trajectories along the $X$ axis. The second plot depicts the angle of the platform and the third depicts the paths followed by the platform on the $X-Y$ plain.

It is observed that when $\omega_{m d}=1080 \mathrm{rad} / \mathrm{s}$ and $\omega_{m e}=$ $900 \mathrm{rad} / \mathrm{s}$, the platform develops rotational speed larger compared to that when $\omega_{m d}=1060 \mathrm{rad} / \mathrm{s}$ and $\omega_{m e}=950 \mathrm{rad} / \mathrm{s}$. On the other hand, the first set results in linear speed lower compared to that of the second set. This observation is equivalent to saying that a larger difference $\omega_{m d}-\omega_{m e}$ results in a larger rotational speed, whereas a larger mean value $\left(\omega_{m d}+\omega_{m e}\right) / 2$ results in a larger linear speed of the platform. Simulations have showed that this result can be generalized for any combination of actuation speeds in the useful actuation range $\omega^{*}$.

Hence, we see that the asynchronous operation produces two dof motion. At the same time, as shown in the zoom-in plots in Fig. 8, the asynchronous actuation generates successive static and kinetic phases, which correspond to the valleys and peaks 

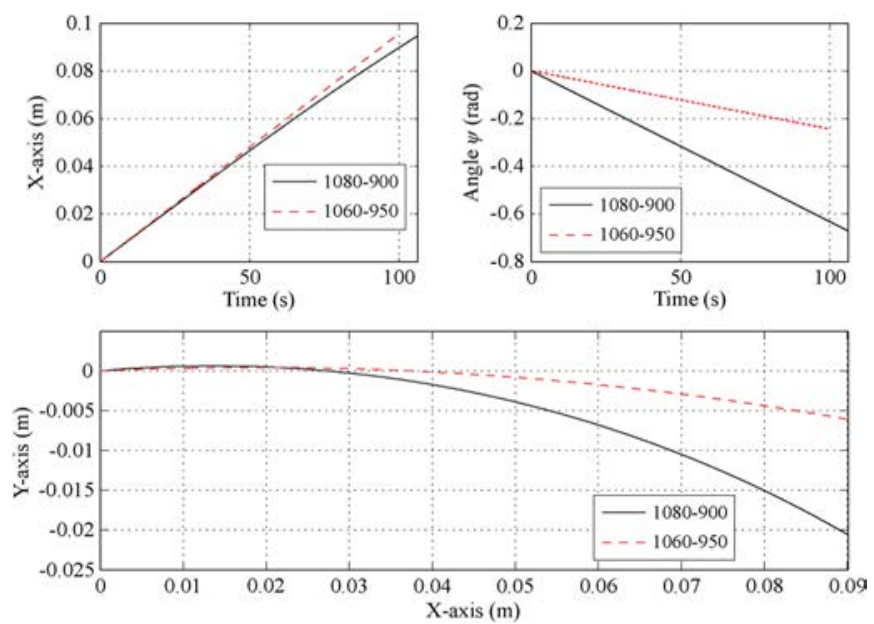

Fig. 7. Two simulation examples that demonstrate the platform response for asynchronous operation. The black solid line represents motion due to the first set and the red dashed line represents the motion due to the second set.
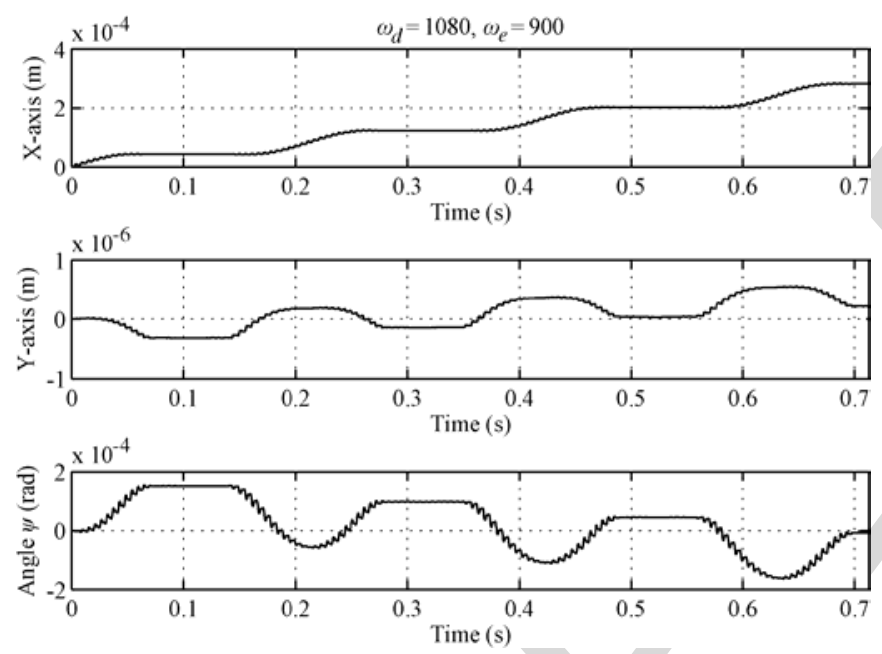

Fig. 8. Zoom in on the $x, y, \psi$, trajectories of the platform.

of the modulating envelop of the sinusoidal beats of the actuation forces and moments. These alternating phases result in oscillatory motions (for all three motion components) that reduce the motion resolution of the platform. Hence, asynchronous operation increases platform mobility from one to two dof at the expense of motion resolution.

Next, we demonstrate how asynchronous operation can be employed to compensate for undesired motion due to platform asymmetries. We consider the case where, due to asymmetry, the $\mathrm{CM}$ of the platform is located at a distance $r=0.002 \mathrm{~m}$ from the centroid and at an angle of $\varphi=30^{\circ}$ with respect to the platform body-fixed $x$ axis.

Fig. 9 depicts the trajectories of the asymmetric platform for the case of synchronous operation. Fig. 10 demonstrates the corrective action of the asynchronous actuation, i.e., $x$-translation is maintained, while undesired $y$-translation and rotation have been reduced by one and two orders of magnitude respectively. Therefore, we see that small manufacturing asymmetries can be compensated by proper driving of the two micromotors.
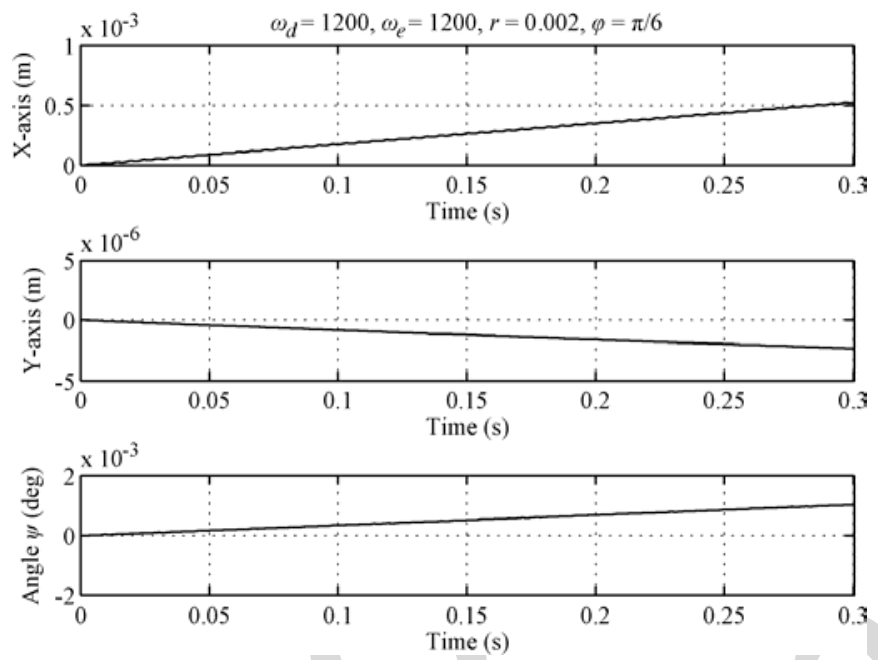

Fig. 9. Synchronous operation. The platform exhibits parasitic displacement and rotation along the $Y$ axis and about $Z$ axis, respectively.
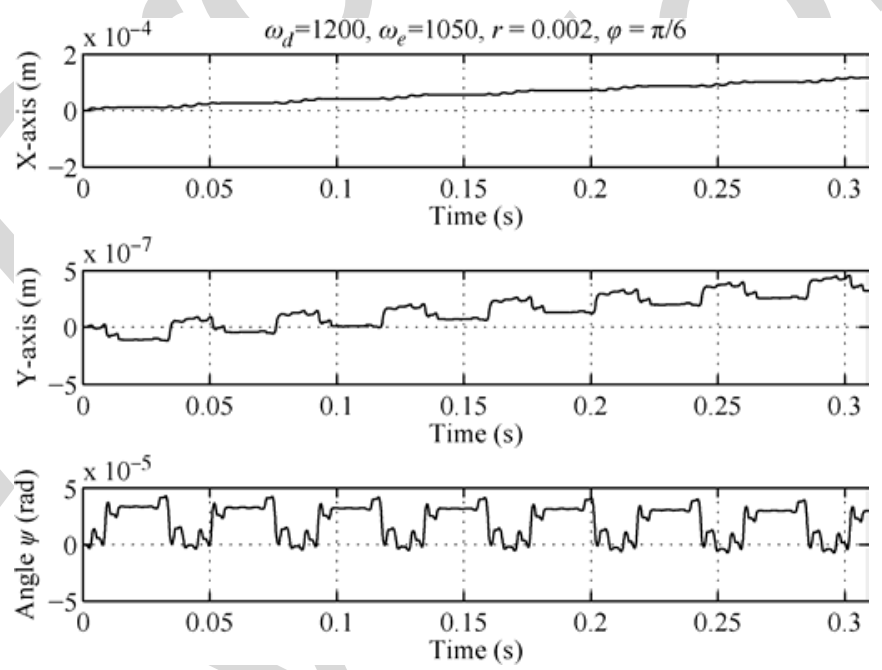

Fig. 10. Asynchronous operation compensates for the parasitic motion due to the platform asymmetry.

\section{Controller Design}

In this section, we focus on the development of a closed-loop motion controller that drives the microrobot end-effector along a desired trajectory towards a desired goal position $\left[x_{d e s}, y_{d e s}\right]^{T}$. The tip of a needle mounted on the microrobot represents the end-effector.

Based on the platform open loop motion, a good choice for the system control inputs would be the vector $\left[\omega_{m d}, \omega_{m e}\right]^{T}$. Accordingly, a simple controller would be two PDs; one for adjusting the mean value of motors speeds and the other for adjusting their difference. This way the two dof of the robot would be controlled as explained in the previous section. However, owing to system complexity and hardware limitations, the actual region within which the motors operate is a subspace of the theoretical useful actuation range $\omega^{*}$. This means that the available control authority is bounded, and the PD controllers drive the motors to saturation resulting in large trajectory tracking errors. 


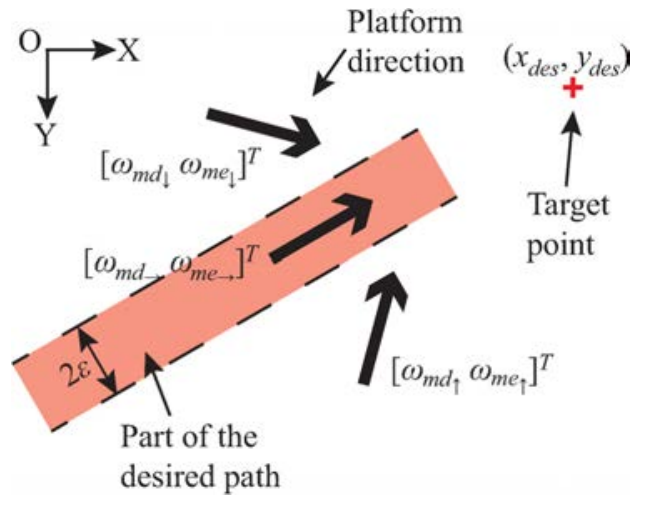

Fig. 11. Graphical representation of the proposed controller action.

To address this issue, a simple rule-based controller has been designed that takes into account these limitations. It is described by the following set of rules:

$$
\left[\begin{array}{c}
\omega_{m d} \\
\omega_{m e}
\end{array}\right]= \begin{cases}{\left[\omega_{m d \downarrow} \omega_{m e \downarrow}\right]^{T}} & \text { if } \quad y<y_{\text {des }}-\varepsilon \\
{\left[\omega_{m d \rightarrow \omega_{m e \rightarrow}}\right]^{T}} & \text { if } \quad y_{\text {des }}-\varepsilon<y<y_{\text {des }}+\varepsilon \\
{\left[\omega_{m d \uparrow} \omega_{m e \uparrow}\right]^{T}} & \text { if } y>y_{\text {des }}+\varepsilon\end{cases}
$$

where $\left[\omega_{m d \downarrow}, \omega_{m e \downarrow}\right]^{T}$ and $\left[\omega_{m d \uparrow}, \omega_{m e \uparrow}\right]^{T}$ denote motor angular velocity pairs that result in a platform displacement with a positive or negative instantaneous curvature, respectively. The vector $\left[\omega_{m d \rightarrow}, \omega_{m e \rightarrow}\right]^{T}$ denotes the pair of motor angular velocities that result in straight line translation, and $2 \varepsilon$ designates the width of the acceptable path. The specific angular velocity pair values depend on system parameters and distance from the target, and are identified by experiments.

A graphical representation of the controller action is illustrated in Fig. 11. The colored strip represents part of the desired path. The platform is forced to translate inside the desired path strip. When the needle tip reaches the target location, both motors are stopped.

\section{RoBOt PROTOTYPE}

A microrobot prototype was built, see Fig. 12. This includes two vibration DC motors fed by pic-controlled H-bridges, wireless communications to a PC commanding station, a needle with force sensing capabilities, and an on-board battery. For more information, one can look at [11]. We opted for DC motors because their small size, as well as the small footprint of their driving electronics, allows designing a compact and miniature mobile robot. An alternative option was to employ stepper motors. Their advantage is that they could drive the two motors in synchronization without using closed-loop control. However, stepper motors require complicated and bulky electronics and would not allow for a compact design (for example, the driver board of Faulhaber's ADM 0620 has a surface approximately $83 \times 53 \mathrm{~mm}$ ). Moreover, stepper motors themselves are larger and more expensive.

The experiments presented in the next section are designed by taking into account a microassembly scenario. According to this, a microassembly task consists of two phases. In the first phase, the microrobotic platform executes a macroscale motion towards a target. In the second phase, the platform executes mi-
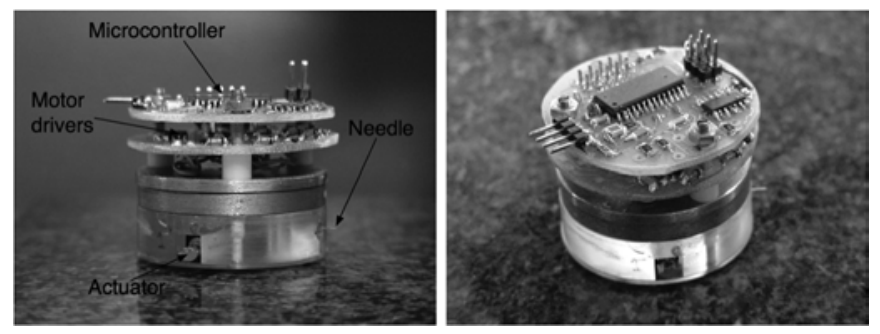

Fig. 12. First prototype of the microrobot: (a) lateral view and (b) angle view.

croscale motions, and the microassembly or micromanipulation task is performed in the field-of-view of a microscope. While the first phase demands increased velocity, the second phase requires increased motion resolution.

\section{OPEN LOOP EXPERIMENTS}

In this section, the results from open loop experiments are given and discussed. These results are used to quantify open loop motion characteristics of the platform such as motion resolution and speed, and to understand its open loop limitations. Furthermore, the knowledge acquired by these experiments is used to implement the closed-loop control architecture presented in Section V. The experiments are implemented by driving the micromotors in synchronous and asynchronous mode. In both modes, the desired angular speed of the motors was selected by trial and error, i.e., the voltages producing the desired speed in each motor were identified and applied. However, measurements showed that the speed of each motor has an error of about $\pm 1 \%-2 \%$, hence limited synchronization errors of the actuators were observed. In this sense, the "synchronous" mode is actually "quasi-synchronous."

\section{A. Macroscale Translational Motion}

In the macroscale experiments, the platform motion is recorded by a digital video-camera. The video file is then processed offline by image processing routines of the Image Processing Toolbox of Matlab. To capture both position and orientation, three white circular marks were added to the top surface of the microrobot.

In the first experiment, the motors operate synchronously at 10000 RPM, with the same sense of rotation. The platform performs a pure translation (as described in Section IV). The resulting microrobot $X-Y$ plane path and trajectory are depicted in Fig. 13. Ideally, the platform should perform pure translational motion along the $X$ axis. In practice the platform translates along the $X$ axis at $1.2 \mathrm{~mm} / \mathrm{s}$, exhibiting an $8 \%$ translation along the $Y$ axis and a $6^{\circ}$ rotation.

The parasitic motion components are due to microrobot asymmetries, errors in actuation synchronization and nonuniform distribution of friction on the supporting surface. These error sources can be significantly reduced by closed-loop control.

\section{B. Macroscale Rotational Motion}

The actuators operate synchronously at 10000 RPM, in an opposite sense, and the platform performs pure rotation. The path and the trajectories are depicted in Fig. 14. 

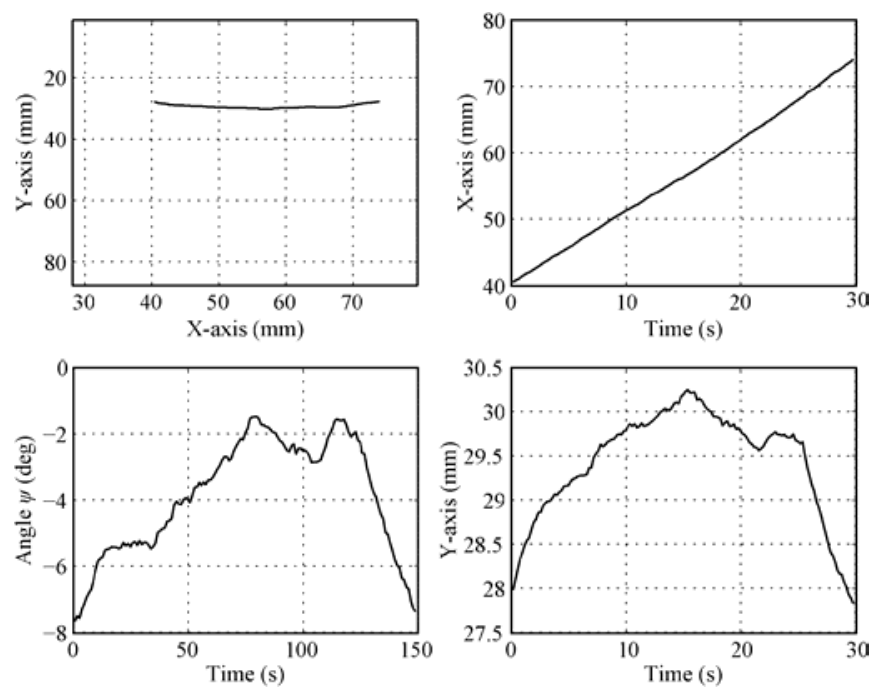

Fig. 13. Macroscale translation of the platform.
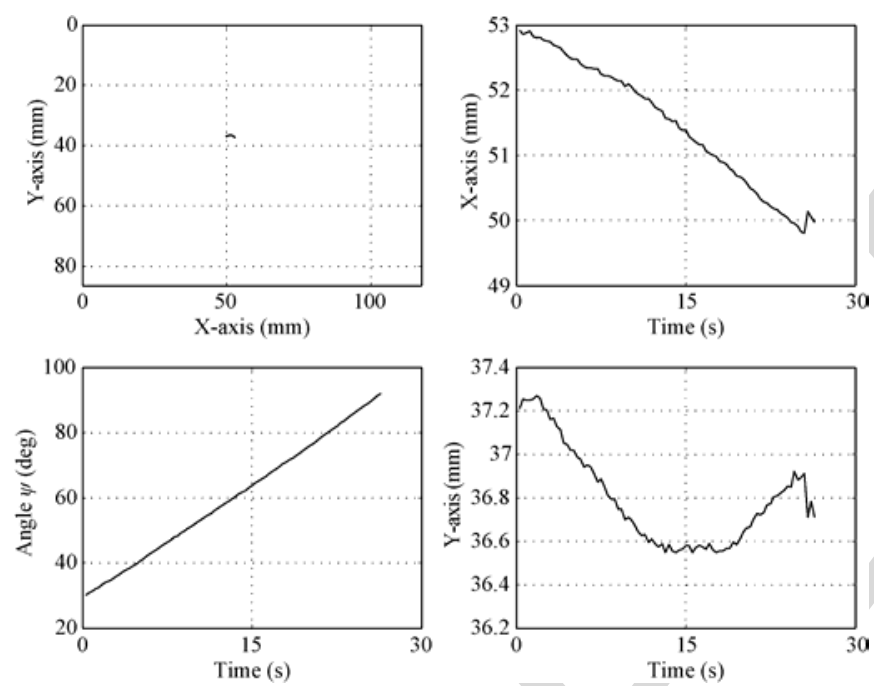

Fig. 14. Macroscale rotation of the platform.

The platform rotates about its vertical axis at a constant speed of $0.052 \mathrm{rad} / \mathrm{s}$. The platform also exhibits open loop translational speed of approximately $0.1 \mathrm{~mm} / \mathrm{s}$ and $0.03 \mathrm{~mm} / \mathrm{s}$ along the $X$ and $Y$ axis, respectively.

\section{Macroscale Combined Motion}

The actuators operate asynchronously at $\omega_{m d}=$ $10000 \mathrm{RPM}$ and $\omega_{m e}=9000 \mathrm{RPM}$. The path and trajectories of the platform are depicted in Fig. 15. The microrobot performs a curvilinear motion on the $X-Y$ plane. The corresponding simulation in Section IV has predicted similar trajectories.

\section{Microscale Translational Motion}

The microscale motion of the platform is measured by following the trajectory of the tip of the needle mounted on the platform. The needle tip motion is recorded by a video-microscope. The video camera pixel size was chosen so that the measurement resolution of the system is approximately $2 \mu \mathrm{m}$. The
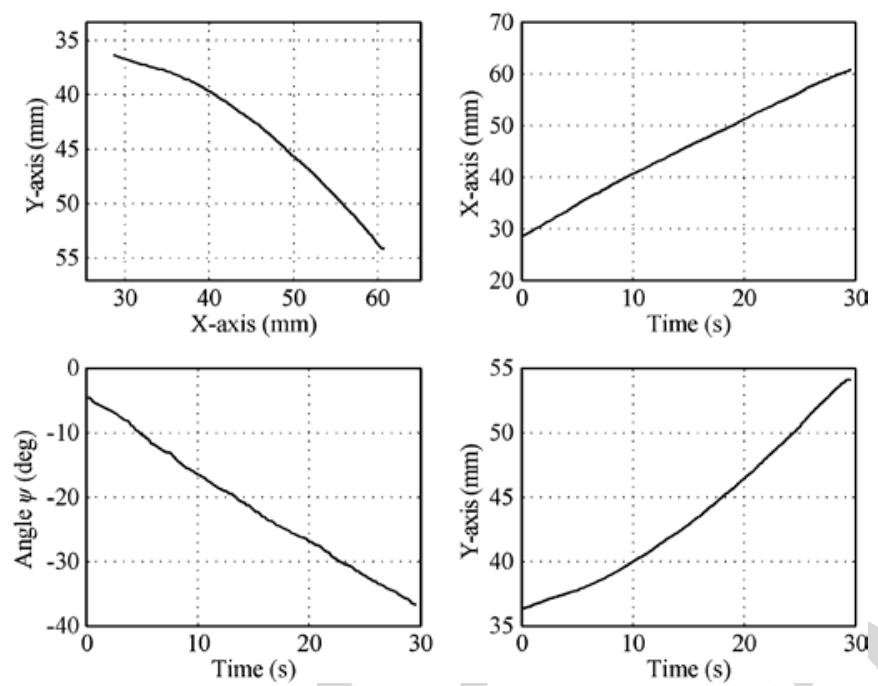

Fig. 15. Macroscale combined motion of the platform.
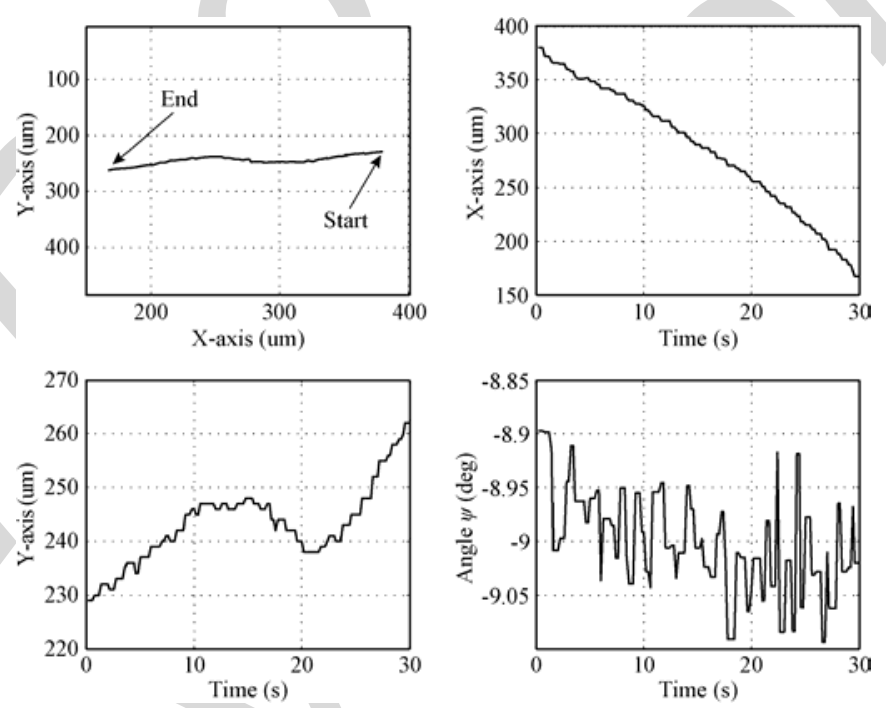

Fig. 16. Microscale translation of the platform: Translation and rotation of the micro-needle tip within the field of view of the microscope.

video camera selected was the Marlin F146B, from Allied Vision Technologies, GMBH.

The actuators operate synchronously at a speed of 7000 RPM and at the same sense of rotation. The microrobot motion response is depicted in Fig. 16. It moves for $215 \mu \mathrm{m}$ along the $X$ axis at a speed of $8 \mu \mathrm{m} / \mathrm{s}$. The microrobot needle tip exhibits undesired translation of $30 \mu \mathrm{m}$ along the $Y$ axis, which is due to a $2 \times 10^{-3} \mathrm{rad} / \mathrm{s}$ angular oscillation of the microrobot about its $\mathrm{CM}$. The angular oscillation is due to synchronization errors of the actuators. The actual $Y$-translation of the $\mathrm{CM}$ of the platform is estimated to be less than $5 \mu \mathrm{m}$. When the actuation command is set to zero (stop command) the platform exhibits a transient response, during which it covers a distance of up to $5 \mu \mathrm{m}$. Incorporating all sources of error leads to an open loop translational microrobot motion resolution approximately $20 \mu \mathrm{m}$. Note that increasing the robot's mass would result in higher motion resolution due to higher friction forces at the contact points, but at the same time this would cause a less efficient motion. A more efficient way to increase the motion resolution would be to choose smaller eccentric mass $m$ (or equivalently 
TABLE II

START, END, AND TARgET PoINT COORDINATES

\begin{tabular}{|c|c|c|}
\hline & X coordinate $(\boldsymbol{\mu} \mathbf{m})$ & Y coordinate $(\boldsymbol{\mu m})$ \\
\hline Start point & 579.3 & 619.3 \\
\hline End point & 1747.6 & 1106.9 \\
\hline Target point & 1745 & 1111 \\
\hline
\end{tabular}
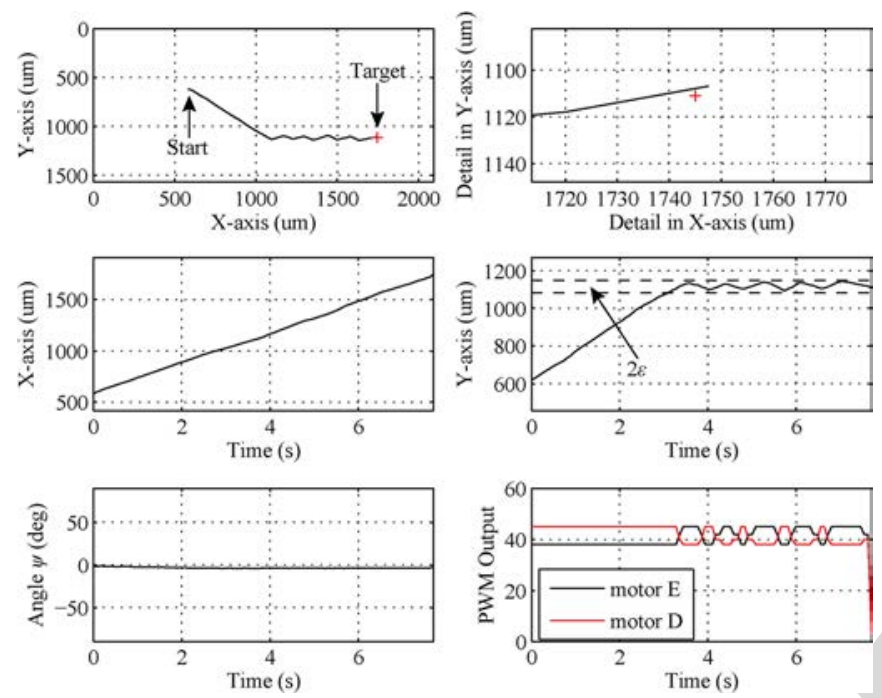

Fig. 17. Microscale positioning experiment: (a) path of the micro-needle tip, (b) zoom in on the end position of the micro-needle tip, (c) $x$ trajectory of the tip, (d) $y$ trajectory of the tip, (e) $\psi$ trajectory of the tip, and (f) PWM output from the controller.

smaller moment arm $r$ ) and a higher actuation speed $\omega_{m}$, so that the step of the platform reduces, i.e., motion resolution increases, while the threshold values in Table I remain the same.

\section{Closed-LoOP CONTROL EXPERIMENTS}

This section presents a set of experimental closed-loop motion results. As mentioned earlier, the second phase of the microassembly scenario requires increased motion resolution. This can be achieved using the controller of Section V. To validate the controller, a number of closed-loop microscale translational motion experiments were conducted. The goal for the microrobot-mounted needle tip is to follow a predefined horizontal corridor-like path of width $2 \varepsilon$, reach a desired target point, and then stop.

While the needle tip motion is recorded by the same videomicroscope as in the open loop microscale experiments, here the images are transmitted via a FireWire 400 port to a Core 2, 2.00 GHz PC laptop, and processed on-the-fly in Matlab. The outcome of the image processing of each frame is the plane position of the needle tip. This information is fed back to the controller, and the control inputs are calculated, according to Section V. The inputs, expressed as PWM commands, are transmitted wirelessly to the microrobot and the appropriate voltages are applied to its motors. The control loop duration is $80 \mathrm{~ms}$.

Table II summarizes the needle tip coordinates of the Start, the End point, and the Target point. As shown in Fig. 17(a), the needle tip begins its motion at the Start point. After $3.1 \mathrm{~s}$
TABLE III

CONTROLler PARAMETERs FOR $\varepsilon=30 \mu \mathrm{m}$

\begin{tabular}{|c|c|c|c|}
\hline$\omega_{\text {md }}$ & PWM \% & $\boldsymbol{\omega}_{\text {me }}$ & PWM \% \\
\hline$\omega_{m d \downarrow}$ & $45 \%$ & $\omega_{m e \downarrow}$ & $38 \%$ \\
\hline$\omega_{m d \rightarrow}$ & $40 \%$ & $\omega_{m e \rightarrow}$ & $42 \%$ \\
\hline$\omega_{m d \uparrow}$ & $38 \%$ & $\omega_{m e \uparrow}$ & $45 \%$ \\
\hline
\end{tabular}
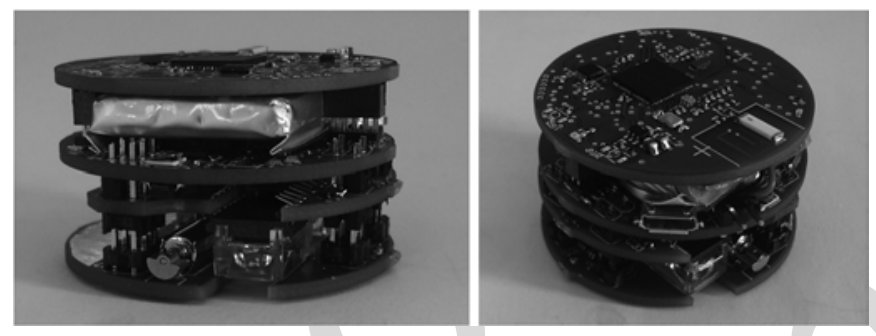

Fig. 18. Second prototype of the microrobot: (a) lateral view and (b) angle view.

it reaches the desired path strip and remains in it, moving towards the Target point. From Fig. 17(b), it can be seen that the position error along the $X$ and $Y$ axes is 2.6 and $4.1 \mu \mathrm{m}$ respectively. The needle tip trajectories along the $X$ and $Y$ axes are depicted in Fig. 17(c) and (d). The orientation of the tip is shown in Fig. 17(e). The commanded angular velocities of the motors are expressed as PWM control values and are presented in Fig. 17(f). Table III summarizes the controller parameters.

As shown by this and other experiments, closed-loop motion control yields a $5 \mu \mathrm{m}$ resolution, i.e., a fourfold improvement compared to open loop operation. The position errors are due mainly to steady-state motor speed discrepancies and to the slow motor speed transient response. It is expected that the addition of motor speed control will reduce further the position error due to an increase in the actuator bandwidth. The experimental results described here are supported by a video, which is submitted with this paper.

\section{CONClusion AND Future Work}

This paper presented the analysis, design, and closed-loop motion control of a mobile microrobotic platform capable of micrometer positioning on a plane. The mobile microrobot, including chassis, actuators, drives, microprocessor, and electronics is of low cost (less than $\$ 20$ ), can be fabricated rapidly and is made of commercially available components. The microrobot motion is induced by centrifugal forces generated by two DC vibration motors installed inside the platform body. The dynamic model of the microrobot platform was developed to predict its motion. Using this dynamic model, the synchronous and asynchronous driving principles were analyzed, simulated and evaluated. The synchronous driving principle provides one dof planar motions of high resolution, either a pure translational or a pure rotational. The asynchronous operation provides two dof motion, but the resulting motion resolution is lower than that of the synchronous operation due to the sinusoidal beat behavior. A controller has been designed to generate controlled planar motion of the platform using a set of rules. Open loop experiments demonstrated that the motion resolution of the 
microrobot prototype is approximately $20 \mu \mathrm{m}$ and its speed is greater than $2 \mathrm{~mm} / \mathrm{s}$. Closed-loop experiments (where the loop closes at the platform position) demonstrated a $5 \mu \mathrm{m}$ motion resolution, i.e. fourfold improvement compared to the open loop experiments. Due to their characteristics, a group of such microrobots can be used in cooperative micromanipulation and/or microassembly tasks in the micrometer scale.

In the future, we plan to experiment with a second prototype that has been built, see Fig. 18. This is characterized by a more compact design, it is equipped with more advanced electronics, and includes additional features, such as optical flow displacement sensors, motor speed optical sensors, and battery recharging through a USB port. The second prototype will give us the opportunity to implement a micromotor speed control scheme, which should improve further the motion performance of the platform.

\section{ACKNOWLEDGMENT}

The authors wish to thank C. Dimitropoulos for his assistance in the realization of the experiments, and A. Nikolakakis for building the second prototype microrobot.

\section{REFERENCES}

[1] S. Fatikow, Ulrich Rembold, Microsystem Technology and Microrobotics, 1 ed. New York: Springer, Feb. 12, 2002.

[2] Y. Sun and B. J. Nelson, "Microrobotic cell injection," in Proc. IEEE Int. Conf. Robot. Autom., Seoul, Korea, 2001, vol. 1, pp. 620-625.

[3] F. Schmoeckel and S. Fatikow, "Smart flexible microrobots for Scanning Electron Microscope (SEM) applications," J. Intell. Mater. Syst. Structures, vol. 11, no. 3, pp. 191-198, 2000.

[4] P. Dario, R. Valleggi, M. C. Carrozza, M. C. Montesi, and M. Cocco, "Microactuators for microrobots: A critical survey," J. Micromech. Microeng., vol. 2, pp. 141-157, 1992.

[5] J. Brufau and M. Puig-Vidal et al., "MICRON: Small autonomous robot for cell manipulation applications," in Proc. IEEE Int. Conf. Robot. Autom., Barcelona, Spain, Apr. 18-22, 2005, pp. 844-849.

[6] A. Bergander, W. Driesen, A. Lal, T. Varidel, M. Meizoso, H. Bleuler, and J.-M. Breguet, "Position feedback for microrobots based on scanning probe microscopy," in Proc. IEEE/RSJ Int. Conf. Intell. Robot. Syst. (IROS'04), Sendai, Japan, 28 Sep.-2 Oct. 2004, vol. 2, pp. $1734-1739$.

[7] S. Martel and I. Hunter, "Nanofactories based on a fleet of scientific instruments configured as miniature autonomous robots," $J$. Micromechatronics, vol. 2, no. 3-4, pp. 201-214, 2004.

[8] A. T. Nguyen and S. Martel, "Locomotion of a miniature robot based on synchronized vibrating actuation mechanisms," in Proc. IEEE/ASME Adv. Intell. Mechatronics (AIM'07), Zurich, Switzerland, Sep. 4-7, , pp. 1-6.

[9] M. Karpelson, G.-Y. Wei, and R. J. Wood, "Driving high voltage piezoelectric actuators in microrobotic applications," Sens' Actuators A: Phys', 2011, J.

[10] P. Vartholomeos and E. Papadopoulos, "Dynamics, design and simulation of a novel microrobotic platform employing vibration microactuators," J' Dynamic Syst., Meas. Control, ASME, vol. 128, pp. 122-134, March 2006.

[11] P. Vartholomeos and E. Papadopoulos, "Analysis, design and control of a planar micro-robot driven by two centripetal-force actuators," in Proc. Int. Conf. Robot. Autom. (ICRA'06), Orlando, FL, USA, May 15-19, 2006, pp. 649-654.

[12] M. Rubenstein, C. Ahler, and R. Nagpal, "Kilobot: A low cost scalable robot system for collective behaviors," in Proc. IEEE Int. Conf. Robot. Autom. (ICRA), 2012[Page range.].
[13] L. Sciavicco and B. Siciliano, Modelling and Control of Robot Manipulators. New York: Springer, 2003.

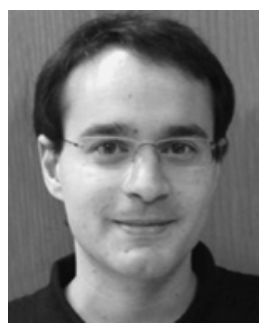

Panagiotis Vartholomeos (S'04-M'08) received the M.Eng. degree in electrical and electronic engineering from Imperial College London, London, U.K., in 2001 and the Ph.D. degree in mechanical engineering/robotics from the National Technical University of Athens, Athens, Greece, in 2007.

From 2008 to 2010, he worked as a Research Associate at the R\&D Department of robotics company ZENON S.A. Since 2010, he has been a Research Fellow at Harvard University and works as a Postdoctoral Researcher with the Cardiac Surgery Bioengineering Laboratory at the Children's Hospital, Boston, MA, USA, and Harvard Medical School, where he conducts research on image guided minimally invasive and ultra-minimally invasive surgical robots. He has participated as a Researcher in the European funded research projects NANOMA and MICRON and as a Senior Researcher and Team Leader in the European Space Agency funded project. His research interests include surgical robotics, microrobotics, control and modeling of dynamic systems.

Dr. Vartholomeos is a Member of the Technical Chamber of Greece. He is the author of more than 25 peer-reviewed publications in journals and conference proceedings.

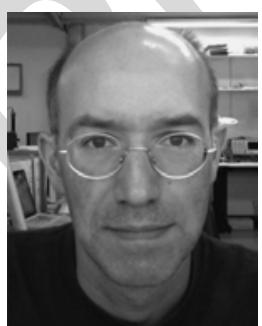

Kostas Vlachos received the B.Sc. degree in electrical engineering from the Technical University of Dresden, Dresden, Germany, in 1993, and the M.S. and Ph.D. degrees in mechanical engineering from the National Technical University of Athens (NTUA), Athens, Greece, in 2000 and 2004, respectively.

From 1996 to 1998, he worked as a Software Analyst. Currently, he is a Postdoctoral Researcher with the Mechanical Engineering Department, NTUA, and Lecturer at the Mechanical Engineering Department, University of Thessaly, where he teaches courses in the areas of control systems, and robotics. His research interests include microrobotics, haptic mechanisms, medical simulation, and control of robotic mechanisms.

Dr. Vlachos is a Member of the Technical Chamber of Greece.

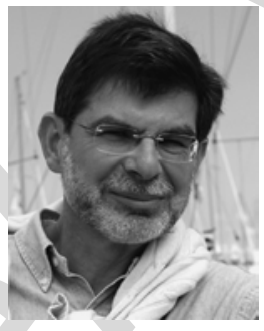

Evangelos Papadopoulos (S'83-M'91-SM'97) received the Diploma degree from the National Technical University of Athens (NTUA), Athens, Greece, in 1981, and the M.S. and Ph.D. degrees from the Massachusetts Institute of Technology (MIT), Cambridge, MA, USA, in 1983 and 1991, respectively, all in mechanical engineering.

He was an Analyst with the Hellenic Navy, Athens, Greece, from 1985 to 1987. In 1991, he joined McGill University, and the Centre for Intelligent Machines (CIM) as an Assistant Professor. Currently, he is a Professor with the Department of Mechanical Engineering, NTUA, where he teaches courses in the areas of systems, controls, mechatronics and robotics. $\mathrm{He}$ has published more than 200 technical articles in journals and refereed conference proceedings. His research interests are in the area of robotics, modeling and control of dynamic systems, mechatronics and design.

Dr. Papadopoulos is a Senior Member of AIAA and a Member of ASME, the Technical Chamber of Greece, and Sigma Xi. He serves as an Associate Editor for the ASME J. of Dynamic Systems, Measurement and Control, and Machine and Mechanism Theory, while previously he served as an Associate Editor of the IEEE TRANSACTIONS ON ROBOTICS, and as a Guest Editor for the IEEE/ASME TRANSACTIONS ON MECHATRONICS. 